\title{
Periodontitis- A Review
}

\section{Aakash Shah*}

Department of Orthodontics and Dentofacial Orthopedics, K.M. Shah Dental College and Hospital, Sumandeep Vidyapeeth University, India

${ }^{*}$ Corresponding author: Shah A, BDS, MDS, Department of Orthodontics and Dentofacial Orthopedics, K.M. Shah Dental College and Hospital, Sumandeep Vidyapeeth University, India, Tel: + 91-9825656377; +91-8200894584; E-mail: aakashshah108@gmail.com

Received date: September 25, 2017; Accepted date: October 07, 2017; Published date: October 20, 2017

Citation: Shah A (2017) Periodontitis- A Review. Med Clin Rev. Vol. 3 No. 3: 14.

Copyright: (C) 2017 Shah A. This is an open-access article distributed under the terms of the Creative Commons Attribution License, which permits unrestricted use, distribution, and reproduction in any medium, provided the original author and source are credited.

\section{Abstract}

The early form of periodontitis is gingivitis where inflammatory changes including bleeding and swelling are restricted to marginal gingiva and surrounding connective tissue, without the involvement of periodontal ligament. Periodontitis occurs when inflammatory changes reach the periodontal ligament and alveolar bone ultimately leading to tooth loss. The underlying cause of the disease is the presence of a polymicrobial biofilm that forms as plaque on the tooth surface and the resulting host response that this plaque induces. Inflammatory responses are initiated by a number of late stage colonizers and the virulence factors they produce. Among these late stage pathogens is $P$. gingivalis, a Gram negative, asacchrolytic bacterium with a large arsenal of virulence factors. $P$. gingivalis produces up to 13 virulence factors involved in host evasion and 21 virulence factors involved in tissue destruction. The presence of these virulence factors induces the production of proinflammatory chemokine and cytokine responses often seen in the saliva and gingival crevicular fluid of individuals with periodontitis.

Keywords: Periodontitis; gingivitis; Risk factors; Host response

\section{Introduction}

Mankind has known periodontitis as a disease entity for more than 5000 years [1]. Clinicians and researchers alike have known that different forms of the disease are in existence with very differing clinical symptoms and rates of disease progression. Chronic periodontitis, a progressive disease affecting gingiva, periodontal ligament and alveolar bone results when gingival inflammation subsequently affects the supporting apparatus of the tooth structure resulting in alveolar bone resorption.

For diagnosis of a disease state, it is important to know the healthy state, any deviation from which will result in disease. In a healthy state, the gingiva is pink or pigmented, stippled, firmly adapted to underlying tissues, scalloped, located at cemento-enamel junction with a 1-3 $\mathrm{mm}$ deep gingival crevice and shows the absence of bleeding on probing. Attached gingiva extends from free gingival groove to mucogingival junction is tightly bound to the underlying bone and resists injury. Transgression from this clinical feature will give an indication of the underlying disease process. The two primary forms of periodontal disease are gingivitis and periodontitis. Gingivitis, the early form disease where inflammatory changes are restricted to marginal gingiva and surrounding connective tissue, without loss of attachment, is a mild and reversible condition.

Periodontitis occurs when inflammatory changes reach the periodontal ligament and alveolar bone and changes are irreversible and destructive, ultimately leading to tooth loss [2].

Apical displacement of the junctional epithelium (JE) results in pocket formation between the tooth surface and gingiva; which is the first indication of destructive disease. It is however known that not all cases of gingivitis progress to periodontitis. Loe in his classical paper on initiation and progression of periodontal disease in Sri Lankan population not exposed to oral hygiene methods found that $8 \%$ of the population had rapid progression of the disease, $81 \%$ had moderate progression of the disease and $11 \%$ of population did not progress beyond gingivitis [3].

Periodontitis itself is classified in various forms and the American Academy of Periodontology classification of 1999 gives a very extensive and exhaustive classification system that encompasses the various disease forms known presently [4].

\section{The prevalence of periodontitis}

Periodontal disease is highly prevalent in the adult US population. The Third National Health and Nutrition Examination Survey (NHANES) that used data from the 2009 to 2010 and 2011 to 2012 cycles showed that $46 \%$ of total adults amounting to 64.7 million people were affected by periodontitis, $8 \%$ of which had a severe form of the disease [5]. Managing and preventing the disease is a colossal task, considering the sheer number of people afflicted with periodontitis. Untreated periodontal patients loose 0.28 teeth 
per patient per year whereas treated patients loose 0.08 teeth per patient per year. Thus periodontal treatment positively affects periodontitis patients by decreasing the tooth loss 3.5 times, a definite indication of benefits of treatment [6].

\section{Risk factors of periodontitis}

A large volume of evidence suggests that certain risk factors associated with periodontal disease may be responsible for differences in the rate of progression of the disease [7]. Periodontitis results from the interplay between the host immune response and bacterial onslaught; factors influencing either of the two may alter the presence and rate of disease progression.

The most popular model of the pathogenesis of periodontal disease by Page and Kornman suggests microbial challenge as an important etiological factor initiating an inflammatory response in susceptible hosts $[8,9]$. The host responds to the invading bacteria with immediate inflammatory and immune response by the production of cytokines and chemokines that cause connective tissue and bone destruction. Also genetic, environmental and various other acquired factors influence this process such that the clinical scenario observed is a summation of all the factors taken together. The disease severity and progression itself can alter the clinical picture by giving a feedback in terms of environment alteration like availability of various nutrients or oxygen [10].

Risk factors like tobacco smoking and diabetes modify the host response to these bacteria, altering the rate and progression of the disease. Diabetes is a metabolic disorder that results in high blood sugar levels over a prolonged period of time and is of two types, Type 1 or Insulin Dependent resulting from failure of the pancreas to produce enough insulin and Type 2 or Non-Insulin Dependent where cells fail to respond to insulin adequately. Gestational diabetics cause high blood glucose levels during pregnancy in women who do not have previously diagnosed diabetes. Diabetes is the result of either a deficiency in endogenous insulin due to the destruction of beta cells or a deficiency of the response to insulin from cells in other tissues. An association between diabetics and periodontitis has been shown unequivocally from the early nineties and most recently in the United States NHANES III where in adults with HbA1c of $>9 \%$ had significantly higher prevalence of severe periodontitis. (OR 2.90, 95\% Cl, 1.40-6.03) [11]. Glycosylated hemoglobin or HBA forms when glucose binds non-enzymatically but irreversibly to hemoglobin. Hemoglobin $\mathrm{A} 1 \mathrm{c}$, or $\mathrm{HbA1c}$, is the major subfraction of $\mathrm{HbA}$ and is considered to be important in reflecting average blood glucose concentration for the past 2-3 months. Maintenance of $\mathrm{HbA} 1 \mathrm{c}$ levels less than $7 \%$ is the goal of diabetics treatment. The cross sectional and longitudinal studies involving the Pima Indian population has shown a 3 fold increase in periodontitis in adults with diabetics [12].

Children with type 1 or insulin dependent diabetics mellitus, resulting from autoimmune destruction of the insulin producing $\beta$ cells of the pancreas, had increased attachment loss and bone loss compared with controls despite similar plaque levels [13]. The association seems to be bidirectional in many studies. One prospective 5 year study of 2,973 nondiabetic individuals demonstrated that those with severe periodontitis had approximately a $5 x$ greater absolute increase in $\mathrm{HbA1c}$ over the observation period when compared to nonperiodontitis patients. Also, some reviews and meta-analyses have identified reductions in $\mathrm{HbA} 1 \mathrm{c}$ following periodontal therapy $[14,15]$.

The interrelationship between smoking and periodontal disease was first observed as early as in 1940s when necrotizing ulcerative gingivitis was seen to be more severe in smokers. However, longitudinal studies, controlling the various confounding factors and proving a direct association came about in early eighties [16]. The NHANES III survey conducted from 1988 to 1994 analyzed 12,329 dentate individuals with respect to their smoking habit and concluded that current smokers were about 4 times as likely as never smokers to have periodontitis (OR 3.97; 95\% Cl, 3.20-4.93), after adjusting for age, gender, race/ethnicity, education, and income: poverty ratio [17]. More recently it was shown that prevalence of periodontitis in smokers is $68.7 \%$ whereas in non-smokers it is $41.4 \%$ for persons above the age 30 [18]. Periodontal cost of smoking is extremely high and is equal to 27 years of disease progression in terms of attachment loss. A 32-year old smoker has the same periodontal attachment loss as a 59-year old non-smoker [19]. A number of factors are responsible for making smoking a significant risk factor in periodontitis patients. Smoking increases the prevalence of anaerobic potential periodontopathogens, chronically reduces the blood flow and vascularity, affects neutrophil function, increases the production of cytokines and chemokines, inhibits fibroblast growth, proliferation attachment and collagen production and decreases immunoglobulin $\mathrm{G} 2$ production. It also reduces the positive effects of surgical and non- surgical periodontal therapy [20].

Other risk factors like genetic variations, cardiovascular disease, stress, obesity, osteoporosis, pregnancy, hormonal alterations have also been associated with increased disease severity [7].

\section{The prevalence of peri-implantitis}

Implants have become a highly predictable alternative for replacement of missing teeth in the last 30 years. In the US alone an estimated of 300,000 to 480,000 implants are placed annually [21]. Branemark revolutionized the field of dentistry with the Osseo integration concept wherein there exists a direct bone to implant contact without any intervening tissue. He defined Osseo integration as "A direct structural and functional connection between ordered, living bone and the surface of a load-carrying implant" [22].

At 16 years, $82.9 \%$ success rates of implants are reported, with a large number of studies quoting the number well above $95 \%$ in 10 years. The multitudinous implants placed for prolonged period of time have increased the incidence of complications like peri- implantitis [23].

Peri-implantitis is an inflammatory condition affecting the peri-implant tissues, involving loss of alveolar bone, whereas 
the inflammatory condition that is limited to the surrounding soft tissues alone is termed as peri-implant mucositis. Periimplantitis and peri-implant mucositis are analogous to inflammatory conditions surrounding teeth viz; gingivitis and periodontitis [24].

When bacteria-induced reversible peri-implant mucositis progresses to involve the surrounding implant supporting bone, the disease becomes irreversible peri-implantitis. Similar to periodontal diseases, peri-implant diseases are also a result of imbalance between the bacterial overload and host immune responses. Increasing probing depth, attachment loss and bleeding on probing are classical signs of both peri-implant and periodontal diseases. Presence of suppuration and radiographic signs of alveolar bone loss may also be present and indicate the progress of disease from mucositis to implantitis. Both diseases usually take a number of years to develop and are painless making long term clinical follow ups and long term prospective studies important in diagnosis and treatment planning.

Patients with periodontal diseases are known to be at increased risk to developing peri- implantitis $[23,24]$. The treatment outcome of peri-implantitis in patients with and without periodontal disease in terms of loss of supporting bone and implant loss are vastly different with significantly increased incidence of peri-implant marginal bone loss in the periodontitis group. Success rate is improved if patients are a part of organized supportive periodontal therapy. A large proportion of anaerobic bacteria like $P$. gingivalis, $P$. intermedia and $F$. nucleatum have been found in the peri-implant sulcus of failing implants as with periodontal pockets emphasizing the need of regular recall maintenance of implants. European Association of Osseo integration accepts an incidence rate of peri-implantitis as one in every five patients.

Patient related modifiable and un-modifiable factors like smoking and diabetics are considered significant risk factors for peri-implant disease. Smoking increases the adverse outcome of implant therapy by an odds ratio of 3.6-4.8. Though implant survival rates are between 80 to $96 \%$ in smokers have been reported, overall they are significantly lower than non-smokers with increased incidence of implant related complications [25]. Although diabetics with an excellent metabolic control are not a contraindication for implant therapy, significantly lower success rates $(68.7 \%)$ of implant treatment and increased risk of peri-implantitis has been reported in diabetics [25]. Continuous hyperglycemia affects a wide range of metabolic pathways and alters the host immune response and wound healing process resulting in impairment of neutrophil adherence, chemo taxis and phagocytosis. This results in persistence of bacterial infection and increased tissue destruction. Hyper responsive macrophages and monocytes increase the proinflammatory cytokines leading to increased inflammation and subsequently alveolar bone resorption.

\section{Virulence factors of $P_{\text {. }}$ gingivalis}

P. gingivalis, a known etiological factor in periodontal disease is frequently isolated from sites infected with periodontitis and is present in higher numbers in areas of active disease. It is a Gram negative asaccharolytic anaerobe, forming black colonies on blood agar and is termed as a pathobiont due to its tremendous destructive capacity. Its arsenal consists of a plethora of virulence factors responsible for its disease producing capacity; prominent among these are the capsular polysaccharide (CPS), lipopolysaccharide (LPS), long and short fimbria, trypsin-like proteases named gingipans and agglutinins, which help it to overcome the host immune responses and invade the host-cells including epithelial, endothelial, and smooth muscle cells [8].

Experimental infections in mice have shown CPS to be a major virulence factor as non- capsulated strains of $P$. gingivalis cause only localized abscesses whereas capsulated strains cause invasive, spreading infections. CPS is also responsible for circumventing host defense mechanisms like opsonization, phagocytosis and complement killing [6]. LPS, found in the outer membrane of Gram negative bacteria is an endotoxin comprising of a lipid $\mathrm{A}$ and a polysaccharide with $\mathrm{O}$ antigen. It is important in enhancing the host inflammatory response leading to initiation and progression of periodontal disease by increasing bone resorption, activation of polyclonal B cells, inhibition of bone formation, and fibroblast proliferation. Immunomodulatory and tissue destroying molecules by fibroblasts and macrophages, and antibodies by lymphocytes are secreted in response to oral LPS leading to progression of periodontal diseases [27]

Fimbriae help the $P$. gingivalis bacteria to attach to the various hosT-cells, a primary step in host cell invasion. They are filamentous and proteinaceous non-flagellar appendages that extend outwards from the bacterial cell [28]. Two distinctly different fimbriae are present on the $P$. ginigvalis cells; major or long, a 49 kDa protein, encoded by fimA gene and minor or short fimbriae composed of $67 \mathrm{kDa}$ protein, encoded by $\mathrm{mfa} 1$ gene. [29]. It has been shown that fimbriae activate both humoral and cellular responses and inactivation of fimA gene reduces periodontal bone loss in gnotobiotic rats.

However the amount of fimbriae produced varies depending on the strains of $P$. ginigvalis, some producing much more than others [30]. Six genotype sequences - type I-V and $\mathrm{Ib}$, are classified depending on the genotype sequence of fimA genes which alters the virulence of the $P$. gingivalis strains, where in type II genotype has the highest (77.80) odds ratio for developing periodontitis [31].

Gingipans are trypsin-like proteinases secreted by $P$. ginigvalis and cause $85 \%$ of proteolytic activity of the bacterium. They are a group of cysteine proteinases encoded by 3 genes $\operatorname{rgpA}, \operatorname{rgp} B$ and $k g p$. They activate the kallikrein/ kinin pathway and increase vascular permeability thus increasing the gingival crevicular fluid. It is also responsible for prostaglandin production and bone resorption, chemotaxis of leucocytes, activation of the clotting system and production of thrombin. Gingipans also degrade fibrinogen suggesting their role in bleeding on probing from periodontal pocket. It impairs the bio- defense cells like neutrophils and monocytes and dysregulates the cytokine network [32]. 
Attachment to the hosT-cells is the first step to invasion of the host and is facilitated by fimbriae and agglutinins. Hemagglutinins are surface proteins that act as adhesins, promoting host colonization by acting as ligands for initial attachment and subsequent invasion [8]. Synthesizing hemagglutinating adhesins establishes the virulence of many bacterial species including Vibrio cholera, Salmonella spp., Bordetella pertussis, and E. coli. P. gingivalis is responsible of agglutination of all erythrocytes of any of the $A B O$ blood groups and also sheep erythrocytes indicating their role in adhesion to the hosT- cells. Five different types of hemagglutinin proteins have been identified and are named as $A, B, C, D$ and $E$. Hemagglutinin $A$ and $B$ are most highly characterized with $A$ and $D$ having $73.8 \%$ homogeniety whereas $B$ and $C$ are $98 \%$ identical. HagB has generated an extensive interest in production of a vaccine against periodontitis and protection against periodontal bone loss with HagB having been successfully tested in a rat model. HagB protein consists of 350 amino acid residues with pl of 8.4 and 49 kDa mass.

HagB is known to induce a robust chemokine and cytokine response from dendritic cells and certain host proteins such as histatins, which belong to the family of anti- inflammatory components of oral cavity, are known attenuate this inflammatory response to HagB [33]. Attachment of bacteria to cells is not restricted to oral tissues, but $P$. gingivalis has been known to invade cardiovascular cells and evidence in form of live bacteria and presence on DNA level has been detected in human atherosclerotic plaques [34].

\section{Biomarkers of inflammation}

In the presence of bacterial components and associated risk factors, the host immune response plays a pivotal role in the disease state. One of the very first occurrences in either gingivitis or periodontitis is vasodilatation of blood vessels below junctional epithelium and migration of neutrophils and monocytes initially, and macrophages and lymphocytes over a period of time. Though the bacterial components initiate these processes the components of inflammation including cytokines and chemokines amplify the process that is manifested as acute and chronic inflammation. Cytokines are cell signaling molecules released in the local environment by host cells affecting the function of other cells. They have an extended spectrum of biological effects on different connective tissue cells, blood vessels, and alveolar bone. The pro- inflammatory and catabolic effects of IL-1 and TNF $\alpha$ results in recruitment of inflammatory cells, vasodilatation of capillaries by enhancing prostaglandin production and alveolar bone resorption by stimulation of osteoclasts and its precursor cells.

Certain cytokines like IL-4, IL-10, IL-11 and TGF $\beta$ are known anti-inflammatory cytokines that can inhibit release of IL-1 and TNF $\alpha$ by targeting the macrophages and producing a programmed cell death [35]. In a 15 year follow up study of cyclic neutropenia, GM-CSF used therapeutically not only increased the number of neutrophils but also improved periodontal disease status with its anti- inflammatory action. Thus cytokines and chemokines have various important pro- inflammatory or anti-inflammatory functions in the host related immune response.

\section{Conclusion}

There are various treatment modalities available for the treatment of periodontitis. A thorough diagnosis and treatment planning is important for the treatment of periodontitis.

\section{References}

1. Highfield J (2009) Diagnosis and classification of periodontal disease. Aust Dent J 54: 11-26.

2. Lindhe J, Lang NP, Karring $T$ (2008) Clinical periodontology and implant dentistry. 5th ed. Oxford ; Ames, lowa: Blackwell Munksgaard.

3. Loe H, Anerud A, Boysen H, Morrison E (1986) Natural history of periodontal disease in man. Rapid, moderate and no loss of attachment in Sri Lankan laborers 14 to 46 years of age. J Clin Periodontol 13: 431-445.

4. Armitage GC (1999) Development of a classification system for periodontal diseases and conditions. Ann Periodontol 4: 1-6.

5. Eke PI, Dye BA, Wei L, Slade GD, Thornton-Evans GO, et al. (2015) Update on Prevalence of Periodontitis in Adults in the United States: NHANES 2009 to 2012. J Periodontol 86: 611-622.

6. Cobb CM (1996) Non-surgical pocket therapy: mechanical. Ann Periodontol 1: 443-490.

7. Knight ET, Liu J, Seymour GJ, Faggion CM Jr, Cullinan MP (2016) Risk factors that may modify the innate and adaptive immune responses in periodontal diseases. Periodontol 2000 71: 22-51.

8. Lamont RJ, Jenkinson HF (1998) Life below the gum line: pathogenic mechanisms of Porphyromonas gingivalis. Microbiology and molecular biology reviews : MMBR 62: 1244-1263.

9. Hajishengallis G, Darveau RP, Curtis MA (2012) The keystonepathogen hypothesis. Nature reviews Microbiology 10: 717-725.

10. Lamont RJ, Hajishengallis G (2015) Polymicrobial synergy and dysbiosis in inflammatory disease. Trends in molecular medicine 21: 172-183.

11. Tsai C, Hayes C, Taylor GW (2002) Glycemic control of type 2 diabetes and severe periodontal disease in the US adult population. Community dentistry and oral epidemiology 30 : 182-192.

12. Taylor GW, Burt BA, Becker MP, Genco RJ, Shlossman M (1998) Glycemic control and alveolar bone loss progression in type 2 diabetes. Ann Periodontol 3: 30-39.

13. Lalla E, Cheng B, Lal S, Kaplan S, Softness B, et al. (2007) Diabetes mellitus promotes periodontal destruction in children. J Clin Periodontol 34: 294-298.

14. Preshaw PM, Alba AL, Herrera D, Jepsen S, Konstantinidis A, et al. (2012) Periodontitis and diabetes: a two-way relationship. Diabetologia 55: 21-31.

15. Vergnes JN (2010) Treating periodontal disease may improve metabolic control in diabetics. Evid-based dent 11: 73-74. 
16. Nociti FH Jr, Casati MZ, Duarte PM (2015) Current perspective of the impact of smoking on the progression and treatment of periodontitis. Periodontol 2000 67: 187-210.

17. Tomar SL, Asma S (2000) Smoking-attributable periodontitis in the United States: findings from NHANES III. National Health and Nutrition Examination Survey. J Periodontol 71: 743-751.

18. Eke PI, Dye BA, Wei L, Thornton-Evans GO, Genco RJ (2012) Prevalence of periodontitis in adults in the United States: 2009 and 2010. J Dent Res 91: 914-920.

19. Haffajee AD, Socransky SS (2001) Relationship of cigarette smoking to attachment level profiles. J Clin Periodontol 28: 283-295.

20. Johnson GK, Guthmiller JM (2007) The impact of cigarette smoking on periodontal disease and treatment. Periodontol 2000 44: 178-194.

21. Paul R, Donald C, David C, Stuart F, Bradley McA, et al. (2013) Peri-implant mucositis and peri-implantitis: a current understanding of their diagnoses and clinical implications. J Periodontol 84: 436-443.

22. Mavrogenis AF, Dimitriou R, Parvizi J, Babis GC (2009) Biology of implant osseointegration. J Musculoskelet Neuronal Interact 9: 61-71.

23. Smeets $R$, Henningsen $A$, Jung $O$, Heiland $M$, Hammacher $C$, et al. (2014) Definition, etiology, prevention and treatment of periimplantitis--a review. Head \& face medicine 10: 34.

24. Heitz-Mayfield LJ (2008) Peri-implant diseases: diagnosis and risk indicators. J Clin Periodontol 35: 292-304.

25. Marcantonio C, Nicoli LG, Marcantonio Jr E, Zandim-Barcelos DL (2015) Prevalence and Possible Risk Factors of Peri-implantitis: A Concept Review. J Contemp Dent Pract 16: 750-757.

26. Brunner J, Scheres N, El Idrissi NB, Deng DM, Laine ML, et al. (2010) The capsule of Porphyromonas gingivalis reduces the immune response of human gingival fibroblasts. BMC Microbiol 10: 5.
27. Wang PL, Ohura K (2002) Porphyromonas gingivalis lipopolysaccharide signaling in gingival fibroblasts-CD14 and Toll-like receptors. Critical reviews in oral biology and medicine: an official publication of the American Association of Oral Biologists 13: 132-142.

28. Yoshimura F, Takahashi K, Nodasaka Y, Suzuki T (1984) Purification and characterization of a novel type of fimbriae from the oral anaerobe Bacteroides gingivalis. J Bacteriol 160: 949-957.

29. Lin X, Wu J, Xie $H$ (2006) Porphyromonas gingivalis minor fimbriae are required for cell-cell interactions. Infect Immun 74: 6011-6015.

30. Sojar HT, Hamada N, Genco RJ (1997) Isolation and characterization of fimbriae from a sparsely fimbriated strain of Porphyromonas gingivalis. Appl Environ Microbiol 63: 2318-2323.

31. Nakano K, Inaba $H$, Nomura $R$, Nemoto $H$, Takeuchi $H$, et al. (2008) Distribution of Porphyromonas gingivalis fimA genotypes in cardiovascular specimens from Japanese patients. Oral Microbiol Immunol 23: 170-172.

32. Imamura $\mathrm{T}$ (2003) The role of gingipains in the pathogenesis of periodontal disease. J Periodontol 74: 111-118.

33. Borgwardt DS, Martin AD, Van Hemert JR, Yang J, Fischer CL, et al. (2014) Histatin 5 binds to Porphyromonas gingivalis hemagglutinin B (HagB) and alters HagB-induced chemokine responses. Sci Rep 4: 3904.

34. Olsen I, Progulske-Fox A (2015) Invasion of Porphyromonas gingivalis strains into vascular cells and tissue. J Oral Microbiol 7: 28788.

35. Birkedal-Hansen H (1993) Role of cytokines and inflammatory mediators in tissue destruction. J Periodontal Res 28: 500-510. 